\title{
La importancia de los factores espaciales y contextuales en la movilidad residencial*
}

\author{
Ricardo Duque-Calvache \\ José Manuel Torrado \\ Nayla Fuster \\ Universidad de Granada \\ ricardoduque@ugr.es; josetr@ugr.es; naylafuster@ugr.es
}

Recepción: 24-01-2017

Aceptación: 04-07-2017

\section{Resumen}

La movilidad residencial es un fenómeno capaz de reconfigurar las ciudades alterando o renovando sus estructuras demográficas y sociales. Pero esta relación entre espacios urbanos y desplazamiento funciona en ambos sentidos, por lo que los contextos también modelan y alteran las movilidades de la población. Los modelos explicativos actuales de los cambios de domicilio parten de una serie de detonantes individuales y de hogar de la movilidad, resultantes de sus características, su estructura y su relación con la vivienda. Nuestro objetivo es medir y evaluar la relevancia de lo espacial una vez controlados estos factores (diferenciando entre dos tipos de movilidad: intramunicipal e intermunicipal) y desentrañar el papel de las diferentes dinámicas internas en el seno de la ciudad metropolitana. En la primera parte, hemos optado por la realización de dos modelos de regresión logística multinomial que ponderan el peso de lo metropolitano en la movilidad individual, para, en un segundo momento, analizar de manera agregada, a través de modelos lineales, qué características de las áreas concretas la explican. El principal descubrimiento de este trabajo es que, independientemente del resto de características individuales, la movilidad residencial es mayor en entornos metropolitanos, especialmente la intermunicipal. Nuestro análisis agregado revela que la movilidad intramunicipal varía entre ciudades según su dinamismo metropolitano; la movilidad intermunicipal, en cambio, está más relacionada con la estructura social, demográfica y del parque de viviendas de cada área concreta, por lo cual es más dependiente en su explicación de los contextos locales.

Palabras clave: movilidad residencial; factores espaciales; áreas metropolitanas; dinámicas urbanas

* Esta publicación forma parte de los resultados del proyecto de investigación «Procesos de reconfiguración social metropolitana» (CSO2014-55780-C3-3-P), del Plan Nacional de $\mathrm{I}+\mathrm{D}$. 


\begin{abstract}
The Significance of Spatial and Contextual Factors for Residential Mobility
Residential mobility is a social force with the capacity to change cities by altering or renovating their social and demographic structures. However, this is a two-sided connection, as the contexts also shape and change the population's decisions on mobility. Current explanations of residential mobility use individual and household variables regarding their characteristics, structures and housing situation. Our goal is to measure the role of spatial variables once we control for the abovementioned factors (classifying the moves into two types: inside and outside the municipality), and to assess the importance of the different internal dynamics of the metropolitan city. In the first part of the paper, two multinomial logit models are used to determine the effect of metropolitan contexts on individual mobility. In the second part, linear models of overall mobility in the metropolitan areas are used to assess their relevant features for residential mobility. The main finding of our research is the measurement of the boosting effect of living in a metropolitan city on residential mobility, after controlling for individual and housing variables (especially in moves out of the municipality). Data on the variations between metropolitan areas allow us to explain the changes in moves inside the municipality, as they are connected to the general dynamism of the city. Moves between municipalities, on the other hand, are linked to the social/ demographic structure and the local housing markets in each area, and therefore have a closer link to the local context.
\end{abstract}

Keywords: residential mobility; spatial factors; metropolitan areas; urban dynamics

\title{
Sumario
}

1. Introducción e hipótesis

2. Marco conceptual

3. Fuentes, población y metodología

4. La relevancia de habitar un contexto metropolitano en la movilidad individual
5. Los factores contextuales y espaciales en la movilidad residencial metropolitana

6. Conclusiones y discusión

Referencias bibliográficas

Anexos

\section{Introducción e hipótesis}

Un análisis sociológico de la movilidad residencial debe partir de la caracterización de esta como una acción social. Ya sea fruto de una decisión personal o colectiva (en el marco del hogar), las circunstancias y los rasgos de los móviles configuran el mapa de los desplazamientos urbanos, que, a su vez, contribuye a redibujar la ciudad y sus espacios. Sin embargo, la movilidad en tanto que acción se enmarca en una estructura que la condiciona: la ciudad, que asume dinámicas y formas metropolitanas (Feria, 2010). La existencia de condicionantes espaciales o contextuales no anulan, pero sí modulan, las decisiones sobre vivienda de individuos y grupos.

El presente trabajo pretende precisamente avanzar en el estudio de la movilidad residencial en España determinando la relevancia de estos factores contextuales en las decisiones individuales, y también desgranando los factores explicativos de esta influencia de lo espacial, de lo metropolitano. Considera- 
mos que es la escala metropolitana la que da sentido y estructura las pautas de cambio de residencia (Susino, 2003), tanto si tienen lugar dentro del mismo municipio - cambios intramunicipales - como entre dos poblaciones diferentes - movilidad intermunicipal.

Partimos de dos hipótesis de investigación

a) Las personas que viven en las áreas metropolitanas se mueven más que aquellas que viven fuera, tanto dentro del municipio como entre municipios diferentes.

b) La movilidad dentro de las áreas metropolitanas se ve condicionada, a su vez, por factores espaciales.

Hemos desarrollado una doble estrategia de explotación de los microdatos censales. En primer lugar, analizaremos el peso de los factores espaciales en la movilidad a través de un modelo de elección individual, como los que convencionalmente se vienen utilizando (Clark y Onaka, 1982; Clark, 2013). La segunda fase se ha llevado a cabo tras crear una base datos, producto de la agregación de los microdatos individuales en áreas metropolitanas, que nos permitirá profundizar en dichos factores espaciales y las variaciones en las tendencias generales.

\section{Marco conceptual}

\section{Movilidad residencial y entorno metropolitano}

Existe un consenso generalizado en torno a los principales factores explicativos de la movilidad residencial, que incluyen fundamentalmente factores individuales y también condicionantes o circunstancias estructurales. La evolución de las aproximaciones al fenómeno de la movilidad ${ }^{1}$ ha ido añadiendo una creciente complejidad y cierta sutileza a modelos inicialmente sencillos. El modelo del ciclo de vida (Clark y Onaka, 1982) y, posteriormente, el enfoque del curso vital (Mulder, 1993) son los grandes hitos en esta trayectoria. En la actualidad, el enfoque del curso vital es la explicación más aceptada y la que se utiliza como referencia en la mayoría de investigaciones (Coulter et al., 2016).

En el caso de España, el interés por el estudio de la movilidad residencial se ha ido consolidando durante las últimas décadas. Los primeros artículos en torno a la cuestión (Aragonés y Amérigo, 1987; Jiménez-Blasco, 1989) tienen carácter exclusivamente teórico, y su interés se centra en divulgar la terminología y los planteamientos teóricos internacionales acerca del fenómeno. Ambos señalan de manera destacada la conexión entre la movilidad residencial y la estructura urbana, esto es, la importancia de los factores espaciales y el contexto local. Décadas más tarde, Módenes-Cabrerizo (2008) señala cómo la relación

1. Que, al mismo tiempo, no dejan de ser investigaciones en torno a la inmovilidad, como acertadamente apuntan Clark et al. (2015), aunque el sedentarismo residencial ha despertado un interés mucho menor que su fenómeno complementario. 
entre el contexto — la definición territorial, según sus palabras_ - y la movilidad funciona como una vía de doble sentido: el entorno modela los desplazamientos de población, pero estos a su vez reconstruyen el espacio habitado. El autor se refiere en este trabajo a la «movilidad espacial», una categorización más amplia que la movilidad residencial (se incluyen también las migraciones, la movilidad cotidiana y otros viajes) que ya había utilizado antes Bericat (1994).

Los primeros trabajos empíricos acerca de la movilidad de la población en España (Díez-Nicolás y Alvira, 1985; Gutiérrez-Puebla, 1992) están mucho más volcados en desplazamientos cotidianos que en los cambios de residencia. El interés por los cambios de vivienda aparece más tarde y está muy vinculado a los estudios centrados en áreas metropolitanas concretas, muy especialmente la de Barcelona (Módenes-Cabrerizo, 1998; Pujadas-i-Rúbies, 2009; Bayonai-Carrasco y Pujadas-i-Rúbies, 2010). Esta conexión entre la preocupación por el estudio de la movilidad residencial y el uso de las áreas metropolitanas como espacio de estudio es interesante y significativa. La movilidad residencial no debería circunscribirse exclusivamente a los desplazamientos dentro de los límites del municipio (considerando migraciones cualquier cambio de residencia fuera de ellos), sino también englobar un espacio más amplio, una ciudad real cuya forma actual es metropolitana (Feria, 2008b). Con posterioridad, se emprenden estudios comparativos entre ciudades (Bayona-i-Carrasco y Pujadas-i-Rúbies, 2014; Feria y Andújar, 2015), pero hasta el momento son pocos los trabajos sobre movilidad residencial metropolitana con datos a escala nacional, con algunas excepciones (Susino y Duque-Calvache, 2013).

$\mathrm{La}$ interdependencia entre factores individuales y espaciales (Brown y Moore, 1970), es decir, entre las características de quienes se mueven y las ciudades metropolitanas en las que lo hacen, debe llevarnos a un análisis de la movilidad que integre los diversos niveles de explicación.

\section{Los factores espaciales en el estudio de la movilidad residencial}

Como afirma Dieleman (2001), la movilidad residencial es un fenómeno complejo que debe entenderse atendiendo a las distintas escalas en las que operan sus factores causales. Dieleman distingue dos niveles en el estudio de la movilidad: uno micro, en el que el objeto de análisis es la elección individual (por parte de personas y hogares), y un segundo nivel meso y macro, en el que se encuentran los factores espaciales y contextuales. Este segundo nivel puede analizarse mediante tres escalas geográficas crecientes: la metropolitana (el mercado local de vivienda), la nacional y la internacional, y en cada una de ellas podemos identificar variables que afectan a la movilidad.

Hay multitud de trabajos que tienen en cuenta alguno de estos niveles y su conexión con diversos fenómenos urbanos. Y no pocos se centran en cómo las áreas metropolitanas influyen en la movilidad. Muchos autores optan por otro término, mercados de vivienda (housing markets), denominación que parece enfatizar los aspectos económicos sobre los demográficos y la preeminencia de las viviendas por encima de sus habitantes. Independientemente de este 
matiz, estos trabajos nos resultan de gran interés, porque, al fin y al cabo, un área metropolitana es, entre otras cosas, un mercado unificado de trabajo y vivienda (Feria, 2008a; Feria, 2010), donde los individuos y los hogares pueden cambiar de domicilio sin cambiar de trabajo, y viceversa. Dicho mercado no es más que el reflejo de la existencia de un espacio de vida colectivo que supera la delimitación municipal (Courgeau, 1988; Susino, 2003).

La relevancia del contexto metropolitano ha sido acreditada en diversas publicaciones. Desde el trabajo de Lu (1998), que ahonda en las diferencias en la movilidad de cuatro regiones de los EE. UU., hasta trabajos que tienen en cuenta los factores espaciales para explicar fenómenos concretos, como las diferencias en la emancipación residencial (Clark y Mulder, 2000), los distintos regímenes de tenencia (Abrahamson et al., 2000), las variaciones en los precios de las viviendas (Malpezzi et al., 1998; Strassman, 2000; Platinga et al., 2013) o las relaciones entre movilidad cotidiana y movilidad residencial (Kim, 2014).

Hay que destacar dos aportaciones fundamentales acerca de las diferencias en la movilidad dentro de las áreas metropolitanas: el trabajo de Dieleman et al. (2000), que analiza las variaciones en la movilidad en 27 áreas metropolitanas estadounidenses, y la publicación de Van der Vlist et al. (2002), que ahonda explícitamente en la relación entre factores espaciales de los mercados de la vivienda y la movilidad. Este último artículo, aparte de contener una excelente revisión teórica y empírica, expone unas conclusiones de gran relevancia. Según estos autores, la movilidad debe entenderse en el marco del mercado inmobiliario en el que se produce, y son cuatro las características fundamentales de estos mercados las que la explican:

- De un lado, las particularidades del parque de viviendas y su tamaño: un mayor parque de viviendas supone una mayor diversidad de opciones $y$, por tanto, potencia un grado más elevado de movilidad. A pesar de ello, esta relación puede entenderse de manera inversa, la movilidad no se amolda a la oferta urbanística, sino que es la movilidad la que genera demanda de nueva construcción (Feria y Andújar, 2015).

- Por otro, el grado de intervención pública en el mercado de vivienda, lo que suelen identificar con el término allocation rules (Van der Vlist et al., 2002). Un mercado de vivienda más intervenido suele relacionarse con una menor movilidad, conclusiones a las que ya llegaron otros autores previamente (Clark y Heskin, 1982; Ault et al., 1994; Munch y Svarer, 2002). Esta variable es menos relevante en nuestro contexto, dado que la vivienda pública tiene un papel prácticamente residual.

- En tercer lugar, destacan las variaciones de movilidad según el grado de urbanización. Sin embargo, las relaciones entre movilidad y urbanización no asumen una relación lineal del tipo «a mayor urbanización, mayor movilidad». La relación puede, de hecho, invertirse, como se constata en el trabajo de Clark y Huang (2003), al aislar el efecto Londres. La movilidad en la capital británica es menor que en el resto del país, a causa de los precios disparados (y disparatados) del mercado inmobiliario. En el contexto 
español, la intensidad y las direcciones de movilidad también varían de manera sustancial en función del grado de desarrollo, de la dimensión y de la estructura de las áreas metropolitanas, e incluso de la situación coyuntural que atraviesan (Feria y Andújar, 2015).

- Por último, y en la línea de otros trabajos, destacan la estructura de los regímenes de tenencia como factor explicativo de la movilidad residencial, correlacionando niveles altos de viviendas en alquiler con tasas elevadas de movilidad, conclusión a la que ya llegaron previamente Dieleman et al. (2000).

En el ámbito nacional, se han realizado también diversos trabajos que han destacado diversos factores territoriales que inciden en la movilidad residencial, como pueden ser el tamaño o la superficie de los municipios (Pujadas-i-Rúbies, 2009) o la cercanía a la cabecera (Bayona-i-Carrasco y Pujadas-i-Rúbies, 2014).

Como vemos, los estudios que tratan la cuestión de los factores espaciales se encuentran dispersos, centrados en temáticas que no siempre se refieren de manera directa a la movilidad. Con frecuencia, estudian otro tipo de relaciones entre las características de las áreas (y/o de los mercados de vivienda) y la movilidad. Además, cabe destacar que, aunque la importancia de los factores espaciales parece ser un presupuesto tácito que no se cuestiona, pocos trabajos han tratado de calibrar de manera explícita en qué medida afectan al número de desplazamientos.

\section{Fuentes, población y metodología}

La fuente utilizada en esta contribución es el Censo de Población y Viviendas 2011, partiendo del archivo de microdatos individuales que el Instituto Nacional de Estadística pone a disposición pública. Entre las principales ventajas que supone emplear esta fuente, encontramos la gran diversidad de variables que contempla, tanto acerca del individuo, como del hogar o de la vivienda. El cuestionario censal ofrece, además, la posibilidad de construir distintas variables referentes a la movilidad, teniendo en cuenta diferentes periodos temporales. En cuanto a los inconvenientes del uso del censo, su carácter de "foto fija» tomada cada diez años dificulta estudiar fenómenos que tengan lugar durante el periodo intercensal, por lo que fuentes con una frecuencia de actualización mayor (como los registros padronales o determinadas encuestas) pueden resultar más adecuadas para estos fines. El censo tampoco recoge información sobre la economía del hogar, muy relevante para los estudios de vivienda.

La elección de la fuente condiciona y ha orientado el diseño de la estrategia de análisis. Los datos permiten analizar los cambios de residencia en el año inmediatamente anterior al censo - 2010 — con respecto al censo anterior (hace 10 años) o el último cambio realizado, independientemente de su fecha. De entre estas opciones, y dado que intentamos acercarnos a los determinantes de la movilidad, hemos decidido tomar la movilidad en el último año para realizar un modelo de regresión logística multinomial. Para este tipo de operaciones estadísticas, lo ideal sería contar con las características de individuos y de hoga- 
res en el momento anterior al cambio, para ver su efecto sobre las decisiones de movilidad. Si bien las variables referentes a las características de los individuos en nuestro estudio se refieren al momento censal, consideramos que la distorsión introducida por la movilidad a un año no es tan problemática, puesto que las características de los individuos no habrán cambiado de manera sustancial.

Otra decisión tomada a partir de las características de la fuente tiene que ver con las restricciones a la desagregación espacial de la información censal de 2011. Los datos individuales no se localizan geográficamente a menos que el municipio de residencia tenga una población superior a 20.000 habitantes. Dado el alto número de municipios menores de ese tamaño que se encuentran integrados en la dinámica urbana metropolitana, ha sido necesario solicitar al INE la reagrupación de los datos, para incluir el total de la población metropolitana independiente del tamaño del municipio de residencia. Para establecer la delimitación de las áreas metropolitanas, se ha seguido el criterio empleado por Feria (2015) a partir de la movilidad entre la residencia y el trabajo.

La población se compone de las personas mayores de 16 años censadas en 2011 que residían en España en 2010. Este planteamiento supone dejar fuera del análisis tanto a los niños y a los adolescentes como a los inmigrantes llegados del extranjero en el año anterior al censo. El hecho de eliminar a los primeros de la muestra responde a un criterio teórico, y es que, al ser un modelo de decisión, consideramos que carece de sentido incluir a una parte de la población que no puede tomar la decisión de moverse, ya que esta no depende de ellos, sino de sus tutores legales. Dicho de otro modo, su movilidad no se explica por sus características personales, sino por las de sus padres, puesto que son movimientos de arrastre (Bayona-i-Carrasco y Pujadas-i-Rúbies, 2010; Bayona-i-Carrasco y Pujadas-i-Rúbies, 2014). Esta decisión puede introducir un pequeño sesgo sobre la imagen conjunta de los procesos de movilidad, ya que los menores de 16 años no se distribuyen por igual en todos los tipos de movimientos, por ejemplo: sabemos que tienen pautas de movilidad específicas (Musterd et al., 2016). También consideramos que las migraciones interiores y la movilidad residencial, que van a ser las dos categorías que nos van a concernir, con especial atención a la segunda, tienen una dinámica diferenciada de las migraciones internacionales, que siguen un patrón propio y complejo (López-Gay y Recaño, 2008; Pujadas-i-Rúbies, 2009; Contreras, 2011). .

Los métodos de análisis empleados se han escogido de acuerdo con las hipótesis que deseamos comprobar. Consideramos que el entorno metropolitano genera unas dinámicas de movilidad más intensas, que van más allá de lo esperable por la mera agregación de una población más joven o de clase más alta que en el espacio no metropolitano. Nos interesa conocer si, estudiando las características personales de los protagonistas de la movilidad residencial, vivir en áreas metropolitanas ocasiona una mayor movilidad. Trabajos previos apoyan esta hipótesis en otros contextos internacionales (Quigley y Weinberg, 1977; Clifford et al., 1982; Kim, 2014), pero es necesario comprobar si esto también se cumple para el caso de la población española, y cuantificar el impacto de lo metropolitano en los desplazamientos. Para ello, se ha utiliza- 
do un modelo de regresión logística multinomial, cuya variable dependiente clasifica a la población a partir de su movilidad en el año anterior al censo. Se han establecido tres categorías: sedentarios, personas que se han mudado pero no han cambiado de municipio (movilidad intramunicipal) y personas que se han movido a un municipio diferente (movilidad intermunicipal). El modelo que construimos es un modelo de decisión, similar a los realizados en otros trabajos (Clark, 2013; Pendakur y Young, 2013; Clark et al., 2015; Kim et al., 2015). Como variables independientes, se han introducido las características personales que han sido identificadas como determinantes de la movilidad en la literatura especializada (relacionadas con el perfil demográfico, la posición social, las características del hogar y la tenencia de la vivienda) ${ }^{2}$. La inmovilidad es la categoría de referencia, por lo que el modelo mide la influencia de las variables independientes utilizadas para participar en uno u otro tipo de movimiento.

El modelo lo realizamos en dos pasos sucesivos, primero incluimos las variables edad, sexo, estructura del hogar, condición sociolaboral (variable que combina la condición socioeconómica y la relación con la actividad y que es aplicable a la totalidad de la población), nivel de estudios y régimen de tenencia de la vivienda. En un segundo paso, se añade una variable binaria referente al lugar de residencia hace un año. En concreto, si hace un año el individuo residía o no en alguna de las áreas metropolitanas existentes en España. Este modelo nos permitirá comprobar si, una vez controladas las características individuales, vivir en un área metropolitana ocasiona una mayor propensión a la movilidad de corto, medio o incluso largo recorrido. Es importante tener en cuenta que este modelo está mezclando dos tipos de cambio de residencia con lógicas diferentes en la movilidad intermunicipal: las migraciones interiores y la movilidad residencial. Tratarlas de manera conjunta puede enmascarar alguna tendencia contradictoria entre ambas, que podría resultar interesante, pero profundizar en esta cuestión se sale de los objetivos del presente trabajo. Esta decisión se ha tomado de manera intencionada, puesto que precisamos disponer de un modelo de decisión sencillo que identifique los factores vinculados a la movilidad de proximidad y la de mayor recorrido, para ello es necesario que toda la población tenga las mismas opciones disponibles - en este caso, quedarse, moverse dentro del municipio o moverse fuera del mismo.

Verificar nuestra segunda hipótesis exige estudiar el comportamiento de la movilidad, ya no para el conjunto de la población española, sino únicamente para la población metropolitana (los que habitaban en ellas en 2011 y también lo hacían en 2010). En esta fase, descartamos, por tanto, las migraciones interiores y solamente contemplamos las dinámicas internas de las áreas. De tal modo, profundizaremos en qué características estructurales influyen en una

2. Algunas de las variables más relevantes, como el grado de hacinamiento (room stress) en la vivienda anterior o los ingresos, no han podido ser utilizadas, dado que la fuente no las proporciona. Sin embargo, consideramos que el modelo que ha podido elaborarse es suficientemente sólido como para establecer comparaciones con investigaciones similares. 
mayor o en una menor movilidad. Para ello, hemos construido una base de datos agregando a los individuos en función de su área de residencia, de manera que nuestras unidades de análisis ya no serían personas, sino las 44 áreas metropolitanas. Las variables en este otro modelo pueden clasificarse en tres bloques principales: variables que definen el conjunto del área, como su extensión, su población total o el incremento de esta; variables sobre la población metropolitana, en términos ocupacionales (tasas de ocupación o actividad), sociales (presencia de grupos sociolaborales) y demográficos (edad media, nacionalidad), y variables referentes al parque de viviendas (volumen y proporción de viviendas vacías, formas de tenencia). Por último, emplearemos variables referentes a la estructura interna de las áreas, en las cuales comparamos las diferencias entre la corona y la cabecera (en cualquiera de los aspectos que acabamos de describir).

Con esta base de datos, es posible analizar las variaciones en la movilidad (tanto intramunicipal como intermunicipal) entre urbes, lo cual nos permitirá contemplar qué factores estructurales hacen aumentar o disminuir los desplazamientos. Puesto que la variable dependiente, la movilidad en un área, es cuantitativa, es posible emplear modelos de regresión lineal simple para identificar y mensurar las variables explicativas clave. Este tipo de estrategia ha sido empleada en trabajos como los de Kim (2014), Van der Vlist et al. (2002) o Cadwallader (1989 y 1992), entre otros. En concreto, se han creado dos modelos, uno para la movilidad intramunicipal y otro para la movilidad intermunicipal, puesto que, como se verá en los resultados, los determinantes de cada una de ellas son diferentes.

El uso del municipio como unidad de referencia es una decisión en parte vinculada a la elección de la fuente de datos, el censo, que usa tal delimitación como elemento clave de su diseño muestral y su presentación de resultados. El mayor problema del uso de los municipios reside en la enorme variedad de tipologías y tamaños que presentan, tanto en las cabeceras metropolitanas como en sus aledaños. Moverse dentro de la localidad no significa lo mismo cuando contamos con términos municipales enormes (como los casos de Zaragoza o Córdoba) que cuando la estructura de asentamientos se basa en unidades reducidas (lo que se da en ciudades metropolitanas como Bilbao o Granada). Algunas encuestas empiezan a contemplar las distancias (medidas espacialmente o por el tiempo que lleva recorrerlas) independientemente de los límites municipales, estrategia que aporta una mayor precisión. En nuestro trabajo, se ha optado por un criterio único (y, por tanto, no adaptado a la casuística particular) para el conjunto del país, porque nuestro interés estriba en conocer la relación, a nivel general, entre el fenómeno de la movilidad residencial y ciertos factores espaciales.

\section{La relevancia de habitar un contexto metropolitano en la movilidad individual}

Un primer análisis descriptivo de las variables nos permite observar variaciones en los perfiles de las personas que participan en las distintas formas de 
movilidad. Esta revisión no solamente tiene un carácter exploratorio, sino que también puede servir para determinar las categorías de referencia en el modelo y anticipar el signo esperado de los coeficientes, que después verificaremos. En términos de edad, se constata que los móviles son más jóvenes que los sedentarios, sin que se perciban diferencias reseñables en las edades medias de los que se desplazan dentro del mismo municipio y entre municipios. La distribución por sexos nos muestra una mayor movilidad de los hombres respecto de las mujeres (Arévalo et al., 2008). Si observamos por separado ambos grupos, se aprecia que el porcentaje de mujeres es ligeramente más elevado en la movilidad intramunicipal que en la intermunicipal, lo cual indica una sutil diferencia por sexos en cuanto al ámbito de movilidad. Las diferencias en la estructura del hogar son notables, puesto que se observa que las familias con hijos a su cargo presentan una menor movilidad que el resto de hogares, y es que, si bien representan más de la mitad de la población, apenas llegan a suponer un tercio de las que cambiaron de domicilio, por lo que la presencia de hijos se muestra como un factor limitante de la movilidad. Si nos paramos a observar las diferencias para cada tipo de hogar, podemos ver que las familias con hijos representan un porcentaje mayor en la movilidad intramunicipal que en la intermunicipal, al igual que ocurre con los hogares unipersonales, y esta relación se invierte en el caso de las parejas sin hijos y de los otros hogares. Es decir, existe una frecuencia mayor de la movilidad de proximidad (intramunicipal) en el caso de las familias y las personas solas. Los extranjeros presentan una mayor movilidad, ya que, pese a que apenas representan una décima parte de la población total, suponen casi un tercio de la población móvil, y este porcentaje es mayor en el caso de la movilidad intramunicipal.

Las variables socioeconómicas muestran un gradiente social más o menos claro, que se muestra especialmente visible en el nivel académico, donde observamos cómo aquellas personas con estudios medios y superiores representan un porcentaje mayor de la población móvil frente a aquellas que tienen estudios secundarios y primarios, y fundamentalmente respecto a las que no tienen estudios. En cuanto a la movilidad por ámbitos, es reseñable ver que los que tienen estudios medios y superiores participan en mayor medida en la movilidad intermunicipal. Si bien el gradiente social es bastante explícito atendiendo al nivel de estudios, no resulta tan intuitivo en el caso de la condición sociolaboral. En términos globales, se aprecia cómo la población activa se mueve más que la inactiva, pero si damos un paso más y nos centramos en la población ocupada (clasificada en profesionales, administrativos, trabajadores de los servicios, operarios y empresarios), podemos ver cómo el citado gradiente social no está tan definido. Si tenemos en cuenta el peso de cada grupo en la población total, podemos apreciar que son los profesionales y los trabajadores de los servicios los que más se mudan, y los empresarios los que menos, cuestión que, con seguridad, está relacionada con el anclaje en el territorio que supone regentar un pequeño negocio, que es precisamente lo más frecuente dentro de este grupo.

El régimen de tenencia también presenta variaciones interesantes entre los móviles intramunicipales y los intermunicipales, más allá de la menor movi- 
Tabla 1. Análisis descriptivo de la movilidad intramunicipal, intermunicipal y el sedentarismo para toda España

\begin{tabular}{|c|c|c|c|c|c|c|c|c|}
\hline \multirow[b]{2}{*}{ Continuas } & \multicolumn{2}{|c|}{ Sedentarios } & \multicolumn{2}{|c|}{ Intramunicipal } & \multicolumn{2}{|c|}{ Intermunicipal } & \multicolumn{2}{|c|}{ Total } \\
\hline & Media & Desviación & Media & Desviación & Media & Desviación & Media & Desviación \\
\hline Edad & 48,3 & 18,7 & 37,2 & 13,8 & 37,0 & 14,6 & 47,3 & 18,6 \\
\hline \multirow[t]{2}{*}{ Edad al cuadrado } & 2.683 & 1.938 & 1.572 & 1.310 & 1.582 & 1.430 & 2.583 & 1.918 \\
\hline & \multicolumn{2}{|c|}{ Sedentarios } & \multicolumn{2}{|c|}{ Intramunicipal } & \multicolumn{2}{|c|}{ Intermunicipal } & \multicolumn{2}{|c|}{ Total } \\
\hline Categóricas & $\mathbf{N}$ & $\%$ & $\mathbf{N}$ & $\%$ & $\mathbf{N}$ & $\%$ & $\mathbf{N}$ & $\%$ \\
\hline \multicolumn{9}{|l|}{ Sexo } \\
\hline Hombre & 17.287.302 & 48,84 & 1.480 .740 & 50,71 & 304.674 & 50,99 & 19.072 .716 & 49,01 \\
\hline Mujer & 18.111.385 & 51,16 & 1.439 .033 & 49,29 & 292.851 & 49,01 & 19.843 .269 & 50,99 \\
\hline \multicolumn{9}{|l|}{ País de nacimiento } \\
\hline Extranjero & 3.658 .918 & 10,34 & 1.042 .963 & 35,72 & 174.212 & 29,16 & 4.876 .093 & 12,53 \\
\hline Español & 31.739 .769 & 89,66 & 1.876 .809 & 64,28 & 423.313 & 70,84 & 34.039 .891 & 87,47 \\
\hline \multicolumn{9}{|l|}{ Estructura del hogar } \\
\hline Unipersonal & 3.732 .606 & 10,54 & 382.489 & 13,10 & 70.129 & 11,74 & 4.185 .224 & 10,75 \\
\hline Pareja sin hijos & 6.680 .031 & 18,87 & 722.494 & 24,74 & 176.696 & 29,57 & 7.579 .221 & 19,48 \\
\hline Familias & 19.414 .908 & 54,85 & 1.069 .664 & 36,64 & 165.706 & 27,73 & 20.650 .278 & 53,06 \\
\hline Otros hogares & 5.571 .141 & 15,74 & 745.125 & 25,52 & 184.994 & 30,96 & 6.501 .260 & 16,71 \\
\hline \multicolumn{9}{|l|}{ Condición sociolaboral } \\
\hline Profesionales & 4.788 .930 & 13,53 & 560.196 & 19,19 & 122.721 & 20,54 & 5.471 .847 & 14,06 \\
\hline Administrativos & 4.340 .795 & 12,26 & 443.975 & 15,21 & 93.656 & 15,67 & 4.878 .426 & 12,54 \\
\hline Servicios & 3.791 .286 & 10,71 & 510.140 & 17,47 & 100.860 & 16,88 & 4.402 .286 & 11,31 \\
\hline Operarios & 5.286 .055 & 14,93 & 548.521 & 18,79 & 101.763 & 17,03 & 5.936 .339 & 15,25 \\
\hline Empresarios & 2.503 .891 & 7,07 & 177.340 & 6,07 & 34.983 & 5,85 & 2.716 .214 & 6,98 \\
\hline Parados & 868.959 & 2,45 & 100.069 & 3,43 & 19.011 & 3,18 & 988.039 & 2,54 \\
\hline Inactivos & 13.471.211 & 38,06 & 542.240 & 18,57 & 115.444 & 19,32 & 14.128 .895 & 36,31 \\
\hline Otros ocupados & 347.560 & 0,98 & 37.291 & 1,28 & 9.088 & 1,52 & 393.939 & 1,01 \\
\hline \multicolumn{9}{|l|}{ Nivel de estudios } \\
\hline Sin estudios & 4.070 .846 & 11,50 & 152.910 & 5,24 & 34.118 & 5,71 & 4.257 .874 & 10,94 \\
\hline Primarios & 5.427 .599 & 15,33 & 311.951 & 10,68 & 59.322 & 9,93 & 5.798 .872 & 14,90 \\
\hline Secundarios & 10.250 .774 & 28,96 & 740.285 & 25,35 & 144.252 & 24,14 & 11.135 .311 & 28,61 \\
\hline FP o bachiller & 9.141 .847 & 25,83 & 949.775 & 32,53 & 194.666 & 32,58 & 10.286 .288 & 26,43 \\
\hline Superiores & 6.507 .622 & 18,38 & 764.852 & 26,20 & 165.167 & 27,64 & 7.437 .641 & 19,11 \\
\hline \multicolumn{9}{|l|}{ Régimen de tenencia } \\
\hline Propiedad pagada & 17.958 .089 & 50,73 & 368.796 & 12,63 & 98.082 & 16,41 & 18.424 .967 & 47,35 \\
\hline Propiedad hipotecada & 11.496 .257 & 32,48 & 917.561 & 31,43 & 180.719 & 30,24 & 12.594 .537 & 32,36 \\
\hline Alquiler & 3.331 .038 & 9,41 & 1.436 .460 & 49,20 & 277.653 & 46,47 & 5.045 .151 & 12,96 \\
\hline Cedida y otras formas & 2.613 .302 & 7,38 & 196.955 & 6,75 & 41.072 & 6,87 & 2.851 .329 & 7,33 \\
\hline \multicolumn{9}{|l|}{ Residía en A.M. en 2010} \\
\hline No & 10.869 .697 & 30,71 & 758.854 & 25,99 & 160.115 & 26,80 & 11.788 .666 & 30,29 \\
\hline Sí & 24.528 .989 & 69,29 & 2.160 .919 & 74,01 & 437.410 & 73,20 & 27.127.318 & 69,71 \\
\hline Total & 35.398 .687 & 90,96 & 2.919 .773 & 7,50 & 597.525 & 1,54 & 38.915 .985 & 100,00 \\
\hline
\end{tabular}

Fuente: elaboración propia a partir de los datos del INE, Censo 2011. 
lidad entre los propietarios, que es generalizada. Por último, y central para el presente trabajo, es el porcentaje que presentan aquellos que hace un año vivían en áreas metropolitanas, frente a aquellos que vivían fuera de estas. Así, aquellos que vivían en áreas metropolitanas representan un porcentaje mayor de la población móvil que de la población inmóvil, siendo esta tendencia inversa en el caso de los que vivían fuera de las áreas, lo cual parece apuntar a que los factores territoriales, y concretamente el hecho de vivir en áreas metropolitanas, provocan que se incremente la probabilidad que los individuos tienen de cambiar de residencia. Si recurrimos a un modelo de regresión logística, podremos determinar si esta relación es estadísticamente significativa o solamente un reflejo de la agrupación de determinados perfiles en el espacio.

\section{Medición del efecto de lo metropolitano mediante modelos de regresión multinomial}

Nuestro primer modelo (ver anexo 1$)^{3}$ es consistente con la literatura sobre movilidad residencial: las variables escogidas son significativas y muestran la relación esperada con ambos tipos de movilidad, tanto por lo visto en el cuadro descriptivo como por trabajos anteriores. En primer lugar, comentaremos las variables independientes una por una; posteriormente, valoraremos el conjunto del modelo y las diferencias entre la movilidad intramunicipal e intermunicipal. La edad (Clark y Huang, 2003), la presencia de hijos en el hogar (Clark y Onaka, 1982) y la inactividad reducen las probabilidades de haberse mudado durante el año anterior, tanto dentro como fuera del municipio (Arévalo et al., 2008; Feria, 2008b). El desempleo, que en otros países origina mayor movilidad (Módenes-Cabrerizo y López-Colás, 2004) y que, en 2004, no ocasionaba cambios significativos, también es un factor que reduce la propensión a desplazarse. Por el contrario, ser extranjero (Duque-Calvache, 2015) y vivir en una vivienda alquilada o cedida son factores que están relacionados con una mayor movilidad (Dieleman et al., 2000). Tanto la ocupación como el nivel de estudios reflejan una gradación en la movilidad, es decir, las personas más formadas y las que desempeñan ocupaciones de clase media son las que más se mueven, tanto en el seno del municipio como fuera de él. La excepción a esta norma la constituyen las personas sin estudios (las que no han alcanzado ningún título o son analfabetas), que presentan una mayor movilidad que la categoría de referencia: estudios primarios. La mayor parte de las personas analfabetas en España tiene edades muy avanzadas, pero una vez descontado el efecto de esta y el resto de variables, la ausencia de títulos tiene un efecto acrecentador de la movilidad, contrariamente a lo esperado (López-Gay y Recaño, 2008) ${ }^{4}$.

3. Se ha preferido dejar el primer modelo en el anexo por su similitud con el modelo 2, del cual solo se diferencia en la inclusión de una variable adicional, en tanto que la mayor parte de los datos se mantienen en gran medida.

4. Cabe plantearse si esa información podría tener conexión con la movilidad forzosa o con la movilidad derivada de la precariedad laboral. Otros trabajos pueden dar mejor cuenta de esa cuestión, por lo que solo apuntamos lo inesperado del dato. 
Además de variables individuales y de hogar, se ha incluido una que tiene carácter agregado: el tamaño del municipio de residencia en 2011 (expresado en escala logarítmica, para evitar las distorsiones debidas a las grandes diferencias en este dato) ${ }^{5}$. Vivir en 2011 en un municipio mayor está relacionado con un grado más elevado de movilidad dentro del mismo (parece lógico que los desplazamientos internos sean mayores si la población dispone de un mercado inmobiliario más amplio). En cambio, para los que viven en los grandes municipios, es menos probable que se hayan mudado desde otra localidad (la dinámica dominante es la salida de habitantes desde grandes núcleos, la suburbanización, y no la centralización, como aprecia Duque-Calvache (2015) para el caso andaluz).

Los datos estadísticos de ajuste del modelo confirman su validez, y aunque su capacidad explicativa no es extraordinariamente alta (como es habitual en los casos de decisión individual, y especialmente si no se cuenta con datos de panel para controlar el efecto inmediato de los cambios vitales), sí supone un punto de partida muy sólido para nuestra investigación. Una vez controlamos las variables individuales de la movilidad, podemos comprobar con precisión si residir en un entorno metropolitano altera la propensión a mudarse y la fuerza de esa asociación. Al añadir la variable, se produce un aumento de la capacidad explicativa (ver anexo 2). Aceptamos, por tanto, el segundo modelo y confirmamos que vivir en un área metropolitana afecta a la movilidad individual. Pero, ¿`con qué intensidad?, ¿hay diferencias entre la movilidad intramunicipal y la intermunicipal?

La nueva variable no distorsiona sustancialmente los resultados del primer modelo. En cuanto a las diferencias entre movilidad interna y movilidad externa al municipio, hay algunas reseñables. Las personas muy mayores muestran un grado más elevado de movilidad intermunicipal, que previsiblemente está conectada en parte con el acercamiento a los hijos (Bayona-i-Carrasco y Pujadas-i-Rúbies, 2014), y esto es coherente con la ausencia de efecto significativo en los cambios intramunicipales: no hace falta acercarse cuando ya se está cerca. Haber nacido en el extranjero se vincula con más fuerza a la movilidad de corto recorrido que a trayectos más largos. La estructura de hogar causa desplazamientos similares en ambos ámbitos, salvo en la variable Otros hogares, que, por su propia definición como conjunto diverso que agrupa situaciones muy distintas, es difícilmente interpretable. El efecto de las categorías sociolaborales y de los estudios es similar, aunque en este caso es más sencillo explicar el cambio de signo de los Otros ocupados, por la importancia en ella

5. Se ha incorporado la variable puesto que hemos empleado la base de microdatos en bruto, sin elevar los casos por el factor correspondiente para ajustar las cifras al total de población en el país. Por este motivo, junto con la exclusión de los menores de 16 años y los llegados del extranjero, nuestra muestra se compone de menos de 4 millones de individuos. Dado que, en la selección de la muestra, desempeñó un papel esencial el tamaño del municipio (en el diseño de la operación censal, se sobrerrepresentan los menores frente a los más grandes), controlar su efecto - combinado con el del resto de variables - nos permite evitar unas conclusiones sesgadas, con lo que podemos mantener un modelo de decisión individual sin ponderaciones. 
Tabla 2. Modelo final de regresión logística multinomial según las características individuales para la movilidad intramunicipal y la intermunicipal

\begin{tabular}{|c|c|c|c|c|}
\hline & \multicolumn{2}{|c|}{ Intramunicipal } & \multicolumn{2}{|c|}{ Intermunicipal } \\
\hline & $\beta$ & Desv. estándar & $\beta$ & Desv. estándar \\
\hline Edad & $-0,036^{\star \star *}$ & 0,001 & $-0,068^{\star \star *}$ & 0,002 \\
\hline $\mathrm{Edad}^{2}$ & 0,000 & 0,000 & $0,000^{\star \star *}$ & 0,000 \\
\hline \multicolumn{5}{|l|}{ Sexo (ref.: Mujer) } \\
\hline Hombre & 0,010 & 0,005 & 0,020 & 0,011 \\
\hline \multicolumn{5}{|l|}{ País de nacimiento (ref.: España) } \\
\hline Extranjero & $0,627^{\star \star \star}$ & 0,007 & $0,398^{\star \star *}$ & 0,015 \\
\hline \multicolumn{5}{|l|}{ Estructura del hogar (ref.: Unipersonal) } \\
\hline Pareja sin hijos & $0,062^{\star \star \star}$ & 0,009 & $0,412^{\star \star \star}$ & 0,019 \\
\hline Familias & $-1,060^{\star \star \star}$ & 0,008 & $-1,057^{\star \star *}$ & 0,019 \\
\hline Otros hogares & $-0,313^{\star \star \star}$ & 0,009 & $0,322^{\star \star \star}$ & 0,019 \\
\hline \multicolumn{5}{|l|}{$\begin{array}{l}\text { Condición sociolaboral } \\
\text { (ref.: Directivos, profesionales y técnicos) }\end{array}$} \\
\hline Personal administrativo y comercial & $-0,062^{* \star *}$ & 0,010 & $-0,042^{* *}$ & 0,019 \\
\hline Resto de personal de servicios & $-0,033^{\star \star}$ & 0,010 & $0,059^{\star \star \star}$ & 0,021 \\
\hline Operarios & $-0,136^{\star \star \star}$ & 0,010 & $-0,194^{\star \star \star}$ & 0,021 \\
\hline Empresarios & $-0,173^{\star \star \star}$ & 0,012 & $-0,267^{\star \star \star}$ & 0,025 \\
\hline Otros ocupados & $-0,062^{\star \star}$ & 0,024 & $0,104^{\star *}$ & 0,045 \\
\hline Parados & $-0,582^{\star \star \star}$ & 0,018 & $-0,621^{\star \star \star}$ & 0,036 \\
\hline Inactivos & $-0,548^{\star \star \star}$ & 0,010 & $-0,583^{\star \star \star}$ & 0,021 \\
\hline \multicolumn{5}{|l|}{ Nivel de estudios (ref.: Primarios) } \\
\hline Sin estudios & $0,094^{\star \star *}$ & 0,013 & $0,125^{\star \star \star}$ & 0,027 \\
\hline Secundarios & $0,058^{\star \star \star}$ & 0,009 & $0,093^{\star \star *}$ & 0,019 \\
\hline FP, bachiller o equivalente & $0,191^{\star \star \star}$ & 0,009 & $0,295^{\star \star \star}$ & 0,019 \\
\hline Superiores & $0,368^{\star \star \star}$ & 0,011 & $0,558^{\star \star *}$ & 0,022 \\
\hline \multicolumn{5}{|l|}{ Régimen de tenencia (ref.: Propiedad) } \\
\hline Alquiler & $1,744^{\star * *}$ & 0,007 & $1,817^{\star \star *}$ & 0,013 \\
\hline Cedida & $0,416^{\star \star \star}$ & 0,009 & $0,324^{\star \star *}$ & 0,019 \\
\hline Tamaño del municipio de residencia & $-0,003$ & 0,001 & $-0,275^{\star \star \star}$ & 0,003 \\
\hline Residía en A.M. en 2010 & $0,058^{\star \star \star}$ & 0,007 & $1,054^{\star \star \star}$ & 0,014 \\
\hline Constante & $-1,158^{\star \star \star}$ & 0,027 & $-0,270^{\star \star \star}$ & 0,053 \\
\hline \multicolumn{5}{|c|}{ Nivel de significación: ${ }^{*} p<0,1,{ }^{* \star} p<0,05,{ }^{\star * *} p<0,01$} \\
\hline \multicolumn{5}{|l|}{ Datos estadísticos de ajuste } \\
\hline Total casos $(\mathrm{N})$ & 3483473 & & & \\
\hline Log. máxima verosimilitud (modelo vacío) & -899185 & & & \\
\hline Log. máxima verosimilitud (modelo final) & -757858 & & & \\
\hline $\mathrm{Chi}^{2}$ & 1515716 & & & \\
\hline $\mathrm{R}^{2}$ ajustado & 0,157 & & & \\
\hline AIC & 1515808 & & & \\
\hline $\mathrm{BIC}$ & 1516409 & & & \\
\hline
\end{tabular}

Fuente: elaboración propia a partir de los datos del INE, Censo 2011. 
de los militares, un colectivo de muy alta movilidad de larga y media distancia. También los trabajadores de los servicios muestran una movilidad mayor que los profesionales, aunque esto solo sucede en cambios intermunicipales. Este grupo se ve sometido a unas condiciones crecientemente precarias, pese a lo cual (o, tal vez, precisamente por ello) tiene una movilidad muy elevada. Este dinamismo, sumado al gran volumen de ocupados que engloba, va a ser importante para la configuración de las áreas metropolitanas españolas, como posteriormente se reseñará.

Finalmente, nuestro mayor interés estribaba en el efecto de lo metropolitano (y su interacción con el tamaño de las poblaciones). Dicho tamaño no altera la movilidad intramunicipal una vez tenemos en cuenta la pertenencia a un área metropolitana, que, a su vez, sí aumenta levemente el desplazamiento de corta distancia. En cuanto a la movilidad intermunicipal, en ella, la pertenencia a un área metropolitana muestra una relevancia muy marcada. Es más probable participar en la movilidad intermunicipal si se vive en un área metropolitana.

Esa aseveración, que podría parecer evidente en tanto que se han definido las áreas como espacios de mayor movilidad, no lo es tanto si tenemos en cuenta lo que aportan los modelos multivariantes. Estos resultados demuestran que el factor espacial, es decir, vivir en un entorno determinado (en este caso, el metropolitano) tiene efectos que no desaparecen al controlar los determinantes individuales de la movilidad. Sabemos que las áreas metropolitanas están menos envejecidas, congregan a población con más estudios, con mejores posiciones socioeconómicas y más personas nacidas en el extranjero, factores que aumentan la movilidad; pero, aun cuando eliminamos ese factor estructural, lo metropolitano influye. Se ha demostrado de manera empírica que la propensión a la movilidad (o al sedentarismo, si queremos hacer la lectura contraria) de dos personas que tengan exactamente las mismas características demográficas, de hogar, laborales, educativas y de tenencia, varían en función de esta cuestión.

\section{Los factores contextuales y espaciales en la movilidad residencial metropolitana}

Hasta ahora, hemos tratado lo metropolitano en su conjunto, como un conglomerado de espacios que potencian la movilidad (al compararse con el resto del territorio). Este análisis simplifica en extremo la cuestión de manera intencionada, puesto que nos interesaba destacar la importancia de las áreas metropolitanas como unidades de significado para la movilidad (frente a otras más utilizadas como el municipio, cuya importancia se ha mostrado menor). Tocado ese punto, podemos avanzar hacia una visión algo más compleja de estos factores contextuales. Las áreas metropolitanas españolas son diversas en tamaños, estructuras y dinámicas, y aunque comparten ciertos rasgos, también se diferencian en otros. Profundizar en la importancia de los factores espaciales requiere desgranar la información por ciudades, tratando cada área metropolitana como una unidad de significado para la movilidad residencial. Además, 
en adelante solo tendremos en cuenta los desplazamientos internos al área metropolitana, de modo que sea posible analizar específicamente las dinámicas metropolitanas internas (Brown y Moore, 1970; Hanuschek y Quigley, 1978).

Hemos optado por construir dos modelos de regresión lineal simple, el primero trata de explicar la movilidad dentro del mismo municipio, mientras que el segundo ahonda en la explicación de la movilidad intermunicipal. Como veremos, las características estructurales de las áreas que explican estos movimientos, así como la capacidad que tenemos para explicar su variabilidad de un área a otra, son sustancialmente diferentes.

\section{Determinantes de la movilidad intramunicipal}

La movilidad intramunicipal en el seno de las áreas metropolitanas españolas dista de ser homogénea, incluso al expresarla en porcentaje sobre la población total. Las cifras varían entre el 5,35\% en el área de Jaén y el 11,98\% de Benidorm, con la media en el 8,37\% y una desviación típica de 1,61 puntos porcentuales. ¿Qué explica unas diferencias tan señaladas?

Ciertas características del conjunto del área y su población pueden aportarnos algo de luz en torno a la cuestión. Hemos realizado una regresión lineal con el crecimiento anual medio de la población, la proporción de ocupados sobre la población total y la proporción de habitantes nacidos en el extranjero como variables independientes, así como el porcentaje de movilidad intramunicipal metropolitano como variable explicada. El modelo alcanza un ajuste $\left(\mathrm{R}^{2}\right)$ del $62 \%$ (tabla 3). Las tres variables contempladas influyen en la movilidad intramunicipal de manera positiva, y todas ellas están vinculadas a un cierto dinamismo del conjunto del área. Aquellas zonas donde hay una mayor ocupación, más crecimiento en el último decenio y más población nacida en el extranjero, pueden considerarse mercados de vivienda más activos, y en ese contexto es perfectamente lógico que la movilidad de corto recorrido sea más intensa.

Aunque la explicación es bastante buena, un análisis de los residuos y los datos atípicos nos llevan a detectar cuatro áreas con valores extremos que afectan al conjunto del modelo. Son zonas con escaso desarrollo metropolitano, pequenas, con fuertes procesos de expansión reciente, y en los casos de Arona-Tenerife Sur y Benidorm, muy volcadas en el turismo ${ }^{6}$. Consideramos que, sacando estas áreas del análisis, los resultados ganarían en claridad. En el modelo refinado (ver de nuevo la tabla 3), no solo mejora la significación, sino también la bondad de ajuste de la recta de regresión, y la $\mathrm{R}^{2}$ aumenta en casi veinte puntos.

A la vista de los resultados, podemos concluir que la movilidad intramunicipal no se distribuye de manera uniforme en todas las áreas, sino que varía de manera significativa y no explicable por causas aleatorias. En el apartado anterior, posiblemente no se pudo apreciar un mayor efecto de lo metropolitano, ya que, al trabajar con datos para el conjunto del país, las diferencias

6. La tabla incluida en el anexo 3 detalla los test empleados para analizar la desviación de estos casos y su importancia para el modelo (Escobar-Mercado et al., 2009). 
Tabla 3. Resumen de modelos para la explicación de la movilidad intramunicipal metropolitana

\begin{tabular}{|c|c|c|c|c|}
\hline & \multicolumn{2}{|c|}{ Modelo inicial } & \multicolumn{2}{|c|}{ Modelo refinado } \\
\hline & $\beta$ & Desv. estándar & $\beta$ & Desv. estándar \\
\hline Crecimiento anual medio de la población & $0,191^{\star \star}$ & 0,094 & $0,260^{\star \star \star}$ & 0,080 \\
\hline Proporción de ocupados & $0,139^{\star * *}$ & 0,048 & $0,112^{\star \star \star}$ & 0,035 \\
\hline Proporción de nacidos en extranjero & $0,116^{\star \star *}$ & 0,031 & $0,154^{\star \star \star}$ & 0,033 \\
\hline Constante & 0,013 & 0,018 & 0,019 & 0,013 \\
\hline \multicolumn{5}{|c|}{ Nivel de significación: ${ }^{*} p<0,1,{ }^{* \star} p<0,05,{ }^{* \star *} p<0,01$} \\
\hline \multicolumn{5}{|l|}{ Datos estadísticos de ajuste } \\
\hline Total casos $(\mathrm{N})$ & 44 & & 40 & \\
\hline $\mathrm{R}^{2}$ ajustado & 0,62 & & 0,80 & \\
\hline AIC & -267 & & -276 & \\
\hline$\underline{\mathrm{BIC}}$ & -274 & & -283 & \\
\hline
\end{tabular}

Fuente: elaboración propia a partir de los datos del INE, Censo 2011.

por ciudades con características opuestas se compensaban y se difuminaban. Al trabajar con las áreas de manera independiente, hemos identificado tres variables que explican las diferencias entre ellas y que están vinculadas a su dinamismo o al de los mercados locales de vivienda, si se prefiere tal terminología. Cuando el área está en expansión, no solamente se producen llegadas de nuevos habitantes, sino que hay más cambios de vivienda de personas que ya vivían allí con anterioridad. Estudios en ciudades concretas ya detectaron que la presencia de extranjeros (Bayona-i-Carrasco y López-Gay, 2011) y los altos niveles de ocupación (Feria, 2008b) también aumentan la movilidad.

Una última cuestión geográficamente relevante es si las áreas metropolitanas se comportan como mercados unitarios, como plantea Susino (2003), es decir, si en zonas con altos niveles de movilidad en las cabeceras metropolitanas el dinamismo afecta también a las coronas. El gráfico 1 confirma la vincula-

Gráfico 1. Relación entre la movilidad intramunicipal en cabeceras y coronas

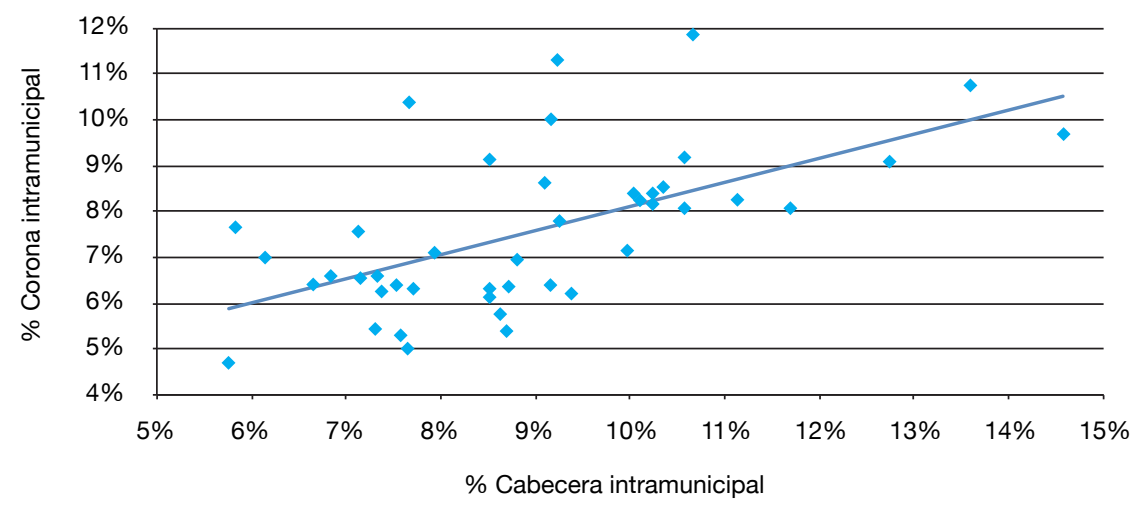

Fuente: elaboración propia a partir de los datos del INE, Censo 2011. 
ción de ambas movilidades y muestra una relación clara entre el porcentaje de movilidad intramunicipal en cabecera y en corona. Hay una fuerte linealidad a lo largo de la diagonal, aunque un buen número de áreas presenta cifras más elevadas en la corona. El dinamismo del mercado residencial afecta a corona y a cabecera, aunque esta asociación varía en intensidad, por lo que podemos considerar las áreas metropolitanas como unidades con cierta coherencia, pero que, al mismo tiempo, poseen tensiones internas.

\section{Las dinámicas intermunicipales en el entorno metropolitano}

En tanto que la movilidad diferencial intramunicipal puede explicarse a través de las variables referentes al dinamismo de las áreas metropolitanas, la explicación de la movilidad intermunicipal es algo más compleja, puesto que interviene en ella la propia composición social de la zona y las características de su parque de viviendas. De modo general, se ha demostrado que vivir en áreas metropolitanas incrementa mucho la probabilidad de participar en la movilidad intermunicipal. Los movimientos de cambio de municipio metropolitano engloban tres flujos muy diferentes: suburbanización, centralización y cambios entre poblaciones de la corona. Son tres fenómenos que marcan las etapas del desarrollo de la ciudad metropolitana, y cuya variabilidad está sujeta a factores contextuales y espaciales.

En la tabla 4, podemos ver las áreas ordenadas según su porcentaje de movilidad total; el reparto de esa movilidad en cambios intramunicipales e intermunicipales, y la composición de los cambios de población en las tres dinámicas principales. La forma más frecuente de movilidad intermunicipal es la suburbanización, que, además, resulta especialmente importante en aquellas áreas en fase de expansión (Susino y Duque-Calvache, 2013). En áreas más maduras, las dinámicas se diversifican. Se incrementan movimientos de centralización y, especialmente, movimientos entre los municipios de la corona que suponen la madurez del área como mercado de vivienda. Cuando estos flujos transversales se consolidan, se generan submercados de trabajo y de vivienda en las coronas.

La interacción entre las dinámicas intermunicipales puede apreciarse en el gráfico 2. A medida que aumentan los niveles de movilidad intermunicipal, esta tiende a complejizarse, de manera que la nube de puntos adquiere una forma cónica, más dispersa cuanto más a la derecha se encuentra. Además, los diferentes tipos de movimientos siguen dos tendencias distintas. A medida que la movilidad intermunicipal aumenta, los flujos de centralización lo hacen en menor medida, con lo que se reduce la dispersión de casos. Los movimientos entre corona y la suburbanización se asemejan entre sí, puesto que muestran una mayor pendiente y una dispersión más acentuada.

Debido a la complejidad que entraña la movilidad intermunicipal, la construcción de un modelo para explicar su variabilidad general en el conjunto de áreas españolas no ha sido fácil, y hemos optado finalmente por una propuesta que, aunque consigue un buen ajuste, tiende a explicar mejor la suburbaniza- 
Tabla 4. Dinámicas de movilidad residencial metropolitana en cifras relativas

\begin{tabular}{|c|c|c|c|c|c|c|}
\hline \multirow[b]{2}{*}{ Área } & \multirow[b]{2}{*}{$\begin{array}{l}\text { Movilidad } \\
\text { total }\end{array}$} & \multicolumn{2}{|c|}{$\begin{array}{c}\% \text { sobre } \\
\text { movilidad total }\end{array}$} & \multicolumn{3}{|c|}{$\begin{array}{c}\text { Composición de la movilidad } \\
\text { intermunicipal }\end{array}$} \\
\hline & & $\begin{array}{l}\text { Intra- } \\
\text { municipal }\end{array}$ & $\begin{array}{l}\text { Inter- } \\
\text { municipal }\end{array}$ & $\begin{array}{c}\text { Hacia } \\
\text { cabecera }\end{array}$ & $\begin{array}{l}\text { Entre } \\
\text { corona }\end{array}$ & $\begin{array}{l}\text { Hacia } \\
\text { corona }\end{array}$ \\
\hline Benidorm & $12,70 \%$ & $94,20 \%$ & $5,80 \%$ & $0,30 \%$ & $3,80 \%$ & $1,70 \%$ \\
\hline Girona & $12,70 \%$ & $91,00 \%$ & $9,00 \%$ & $1,90 \%$ & $3,30 \%$ & $3,80 \%$ \\
\hline Almería-El Ejido & $12,20 \%$ & $91,60 \%$ & $8,40 \%$ & $1,80 \%$ & $2,90 \%$ & $3,50 \%$ \\
\hline Palma de Mallorca & $12,10 \%$ & $92,20 \%$ & $7,80 \%$ & $2,30 \%$ & $2,10 \%$ & $3,40 \%$ \\
\hline Vitoria & $11,20 \%$ & $96,90 \%$ & $3,10 \%$ & $0,90 \%$ & $0,40 \%$ & $1,90 \%$ \\
\hline Madrid & $11,10 \%$ & $88,50 \%$ & $11,50 \%$ & $2,20 \%$ & $5,10 \%$ & $4,20 \%$ \\
\hline Lleida & $10,70 \%$ & $95,80 \%$ & $4,20 \%$ & $0,80 \%$ & $0,70 \%$ & $2,70 \%$ \\
\hline Pamplona & $10,60 \%$ & $87,30 \%$ & $12,70 \%$ & $1,90 \%$ & $3,10 \%$ & $7,70 \%$ \\
\hline Cartagena & $10,40 \%$ & $96,30 \%$ & $3,70 \%$ & $1,50 \%$ & $1,10 \%$ & $1,00 \%$ \\
\hline Málaga-Marbella & $10,40 \%$ & $91,50 \%$ & $8,50 \%$ & $1,50 \%$ & $3,30 \%$ & $3,60 \%$ \\
\hline Tarragona & $10,30 \%$ & $92,10 \%$ & $7,90 \%$ & $0,70 \%$ & $4,80 \%$ & $2,50 \%$ \\
\hline Zaragoza & $10,30 \%$ & $96,90 \%$ & $3,10 \%$ & $1,00 \%$ & $0,30 \%$ & $1,80 \%$ \\
\hline Logroño & $10,10 \%$ & $95,10 \%$ & $4,90 \%$ & $1,50 \%$ & $0,30 \%$ & $3,10 \%$ \\
\hline Barcelona-Sabadell & $10,10 \%$ & $88,10 \%$ & $11,90 \%$ & $2,00 \%$ & $6,10 \%$ & $3,80 \%$ \\
\hline Arona-Tenerife Sur & $10,00 \%$ & $91,80 \%$ & $8,20 \%$ & $4,30 \%$ & $2,00 \%$ & $1,90 \%$ \\
\hline Murcia & $10,00 \%$ & $96,50 \%$ & $3,50 \%$ & $1,50 \%$ & $0,60 \%$ & $1,30 \%$ \\
\hline Toledo & $9,60 \%$ & $90,40 \%$ & $9,60 \%$ & $2,60 \%$ & $3,30 \%$ & $3,80 \%$ \\
\hline Valladolid & $9,50 \%$ & $91,60 \%$ & $8,40 \%$ & $1,70 \%$ & $1,70 \%$ & $5,00 \%$ \\
\hline Castellón & $9,40 \%$ & $94,40 \%$ & $5,60 \%$ & $1,50 \%$ & $1,90 \%$ & $2,30 \%$ \\
\hline Badajoz & $9,00 \%$ & $98,30 \%$ & $1,70 \%$ & $1,10 \%$ & $0,30 \%$ & $0,40 \%$ \\
\hline Elche-Alicante & $8,90 \%$ & $94,90 \%$ & $5,10 \%$ & $0,90 \%$ & $1,60 \%$ & $2,60 \%$ \\
\hline A Coruña & $8,80 \%$ & $88,10 \%$ & $11,90 \%$ & $1,60 \%$ & $4,90 \%$ & $5,50 \%$ \\
\hline Valencia & $8,70 \%$ & $88,90 \%$ & $11,10 \%$ & $2,20 \%$ & $5,10 \%$ & $3,70 \%$ \\
\hline Albacete & $8,70 \%$ & $96,40 \%$ & $3,60 \%$ & $0,30 \%$ & $0,00 \%$ & $3,30 \%$ \\
\hline Granada & $8,50 \%$ & $84,80 \%$ & $15,20 \%$ & $3,60 \%$ & $5,60 \%$ & $5,90 \%$ \\
\hline Pontevedra-Vigo & $8,40 \%$ & $87,50 \%$ & $12,50 \%$ & $2,20 \%$ & $5,30 \%$ & $4,50 \%$ \\
\hline Las Palmas de Gran Canaria & $8,40 \%$ & $89,50 \%$ & $10,50 \%$ & $3,70 \%$ & $3,90 \%$ & $2,90 \%$ \\
\hline Santiago & $8,30 \%$ & $87,40 \%$ & $12,60 \%$ & $2,40 \%$ & $6,00 \%$ & $4,20 \%$ \\
\hline Avilés-Gijón-Oviedo & $8,30 \%$ & $91,50 \%$ & $8,50 \%$ & $2,60 \%$ & $1,40 \%$ & $2,80 \%$ \\
\hline Bilbao & $7,90 \%$ & $86,80 \%$ & $13,20 \%$ & $2,10 \%$ & $7,80 \%$ & $3,30 \%$ \\
\hline León & $7,80 \%$ & $88,20 \%$ & $11,80 \%$ & $3,30 \%$ & $2,70 \%$ & $5,80 \%$ \\
\hline Ourense & $7,80 \%$ & $85,70 \%$ & $14,30 \%$ & $3,70 \%$ & $0,60 \%$ & $10,00 \%$ \\
\hline Salamanca & $7,70 \%$ & $90,70 \%$ & $9,30 \%$ & $2,00 \%$ & $1,40 \%$ & $6,00 \%$ \\
\hline Burgos & $7,70 \%$ & $96,00 \%$ & $4,00 \%$ & $1,20 \%$ & $0,20 \%$ & $2,50 \%$ \\
\hline Santander & $7,70 \%$ & $86,90 \%$ & $13,10 \%$ & $2,70 \%$ & $6,30 \%$ & $4,10 \%$ \\
\hline Huelva & $7,70 \%$ & $95,40 \%$ & $4,60 \%$ & $1,20 \%$ & $1,10 \%$ & $2,30 \%$ \\
\hline Santa Cruz de Tenerife & $7,60 \%$ & $87,30 \%$ & $12,70 \%$ & $2,80 \%$ & $5,20 \%$ & $4,60 \%$ \\
\hline Donostia & $7,60 \%$ & $91,20 \%$ & $8,80 \%$ & $2,40 \%$ & $3,20 \%$ & $3,20 \%$ \\
\hline Córdoba & $7,40 \%$ & $98,00 \%$ & $2,00 \%$ & $0,50 \%$ & $0,20 \%$ & $1,30 \%$ \\
\hline Sevilla & $7,30 \%$ & $92,60 \%$ & $7,40 \%$ & $1,20 \%$ & $2,50 \%$ & $3,70 \%$ \\
\hline Ferrol & $7,20 \%$ & $86,70 \%$ & $13,30 \%$ & $2,80 \%$ & $4,40 \%$ & $6,10 \%$ \\
\hline Algeciras & $7,00 \%$ & $97,50 \%$ & $2,50 \%$ & $0,80 \%$ & $0,60 \%$ & $1,10 \%$ \\
\hline Cádiz-Jerez & $7,00 \%$ & $94,00 \%$ & $6,00 \%$ & $1,70 \%$ & $1,60 \%$ & $2,20 \%$ \\
\hline Jaén & $5,50 \%$ & $96,50 \%$ & $3,50 \%$ & $1,60 \%$ & $0,60 \%$ & $1,30 \%$ \\
\hline Media & $9,10 \%$ & $91,90 \%$ & $8,10 \%$ & $1,90 \%$ & $2,70 \%$ & $3,50 \%$ \\
\hline
\end{tabular}

Fuente: elaboración propia a partir de los datos del INE, Censo 2011. 
Gráfico 2. Proporción de centralización, suburbanización y movilidad intercorona respecto a la movilidad intermunicipal (con rectas de regresión para cada tipo de cambio)

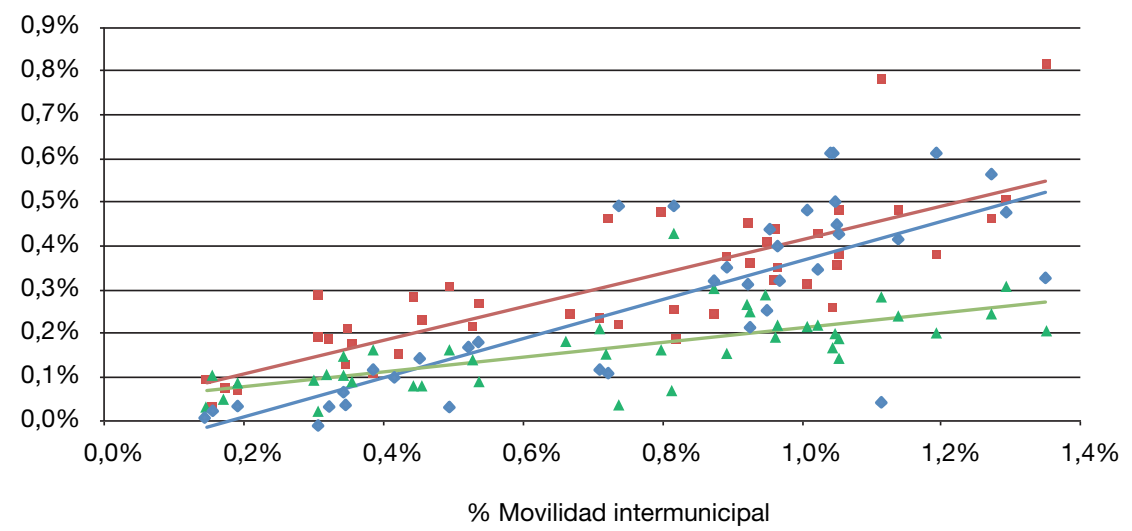

Entre corona

Suburbanización (hacia corona)

$\Delta$ Centralización (hacia cabecera)

Fuente: elaboración propia a partir de los datos del INE, Censo 2011.

ción (que es, al fin y al cabo, la dinámica de movilidad más frecuente) que los otros dos movimientos. La expansión suburbana de las capitales es, además, una fase por la que han pasado o están pasando todas las áreas españolas, en tanto que las dinámicas de movilidad entre municipios de la corona y la centralización ${ }^{7}$ son muy débiles en las áreas poco consolidadas y fuertes en aquellas más asentadas. Es decir, hay una mayor diferencia entre ciudades, lo que dificulta su modelización conjunta.

En la tabla 5, podemos ver los dos modelos realizados. En el primero, se ha intentado replicar el esquema de análisis de la movilidad intramunicipal, recurriendo a las variables que identificamos con el dinamismo urbano. Sin embargo, estas variables no son significativas en esta ocasión. Este es un resultado negativo, pero muy interesante: los determinantes contextuales de la movilidad intramunicipal y la movilidad intermunicipal no son los mismos. Las áreas que más crecen tienen más población nacida en el extranjero y más habitantes ocupados, y no presentan niveles significativamente mayores de

7. La centralización tiene, en sí misma, una naturaleza dual y contradictoria: el mismo tipo de movimiento puede tener significados diametralmente opuestos. En áreas muy incipientes, poco integradas en la dinámica metropolitana, un flujo de centralización puede indicar que la ciudad sigue en la fase de atracción de población de sus alrededores, es decir, en la fase de urbanización, si aceptamos la idea de los ciclos de urbanización (Cheshire, 1995; Champion, 2001). En cambio, en áreas metropolitanas consolidadas, los traslados al centro marcan la fase de reurbanización tras el vaciamiento previo. La dinámica de la centralización es la que más puede beneficiarse de un estudio más centrado en casos concretos, ya que la generalización forzosamente está cubriendo con un manto de homogeneidad una realidad contradictoria. 
Tabla 5. Resumen de modelos para la explicación de la movilidad intermunicipal metropolitana

\begin{tabular}{|c|c|c|c|c|}
\hline & \multicolumn{2}{|c|}{ Modelo inicial } & \multicolumn{2}{|c|}{ Modelo final } \\
\hline & $\beta$ & Desv. estándar & $\beta$ & Desv. estándar \\
\hline Crecimiento anual medio de la población & $-0,035$ & 0,030 & 0,025 & 0,026 \\
\hline Proporción de ocupados & $0,029^{*}$ & 0,015 & $-0,030^{*}$ & 0,017 \\
\hline Proporción de nacidos en el extranjero & 0,013 & 0,010 & $0,021^{\text {** }}$ & 0,010 \\
\hline Extensión del área & & & 0,001 & 0,001 \\
\hline Edad media de la cabecera & & & $0,001^{\star \star \star}$ & 0,000 \\
\hline Proporción de profesionales en cabecera & & & $0,042^{\star \star}$ & 0,020 \\
\hline Proporción de viviendas vacías en corona & & & $0,004^{*}$ & 0,002 \\
\hline Constante & $-0,004$ & 0,006 & $-0,034^{\star \star \star}$ & 0,010 \\
\hline \multicolumn{5}{|c|}{ Nivel de significación: ${ }^{*} p<0,1,{ }^{* *} p<0,05,{ }^{* * *} p<0,01$} \\
\hline \multicolumn{5}{|l|}{ Datos estadísticos de ajuste } \\
\hline Total casos $(N)$ & 44 & & 44 & \\
\hline$R^{2}$ ajustado & 0,16 & & 0,60 & \\
\hline AIC & -374 & & -399 & \\
\hline BIC & -367 & & -385 & \\
\hline
\end{tabular}

Fuente: elaboración propia a partir de los datos del INE, Censo 2011.

cambios de domicilio entre municipios, por lo que podemos afirmar que la movilidad entre municipios tiene más que ver con la estructura interna del área metropolitana, y no tanto con su situación general.

Para mejorar nuestra explicación de estas movilidades, se han introducido nuevas variables referentes a las características de las áreas. El ajuste es bastante mayor (alcanzamos una $\mathrm{R}^{2}$ del 60\%, frente al 16\% anterior), pero, sin embargo, no hemos conseguido alcanzar los niveles del modelo sobre movilidad intramunicipal, ni siquiera añadiendo cuatro variables más. En primer lugar, cabe señalar que la extensión de las áreas (que depende del número de municipios que engloban y su tamaño) no afecta significativamente a la movilidad intermunicipal. En cambio, sí lo hacen las características de cabecera y corona. Las áreas cuyas cabeceras están más envejecidas y tienen un perfil ocupacional más alto presentan más movilidad. Estos datos apuntan a que la movilidad intermunicipal depende de la «madurez» de las áreas, y es mayor en las que ya han pasado por una fase de suburbanización anterior, que típicamente supone la salida de población joven (Galiana y Vinuesa, 2012) y de clase trabajadora (López-Gay y Recaño, 2008). Esos movimientos dejan una ciudad central envejecida y de mayor estatus. Las peculiaridades del mercado local de vivienda también tienen su repercusión en los flujos de movilidad, que se acrecientan cuando las viviendas vacías están localizadas en la corona. Cuando hay un abanico mayor de viviendas disponibles en la corona, la movilidad se diversifica. Es importante no establecer una conexión simplista donde la oferta de vivienda es causa, y la movilidad, efecto. Feria y Andújar (2015) advierten que la causalidad puede revertirse. 
Por último, al introducir las nuevas variables en el modelo, la nacionalidad cobra significatividad, por lo que deducimos que su efecto se hallaba oculto por su conexión con alguna de las otras variables. Los extranjeros constituyen un grupo de alta movilidad, pero, dadas las grandes variaciones en esta variable (la proporción de nacidos en el extranjero en las áreas metropolitanas oscila entre el 3 y el 37\%), posiblemente el efecto conjunto se difumina. Un análisis pormenorizado área por área permitiría detectar casos donde su relevancia es mucho mayor, como se ha demostrado para las áreas de Almería y Málaga (Duque-Calvache, 2015) o Barcelona (Bayona-i-Carrasco y López-Gay, 2011).

\section{Conclusiones y discusión}

La mayor parte de los estudios actuales conceptualizan y estudian la movilidad residencial a partir de factores individuales y del hogar, en conexión con los cursos vitales. Recurriendo a datos de panel y fuentes de información de gran calidad, se ha conseguido profundizar enormemente en refinar los efectos de los cambios familiares, ocupacionales y otros sucesos en las decisiones de cambio de vivienda o en las fases previas a estas (Coulter et al., 2016). Sin embargo, consideramos que, más allá de estos factores, existen regularidades estructuradas espacialmente, efectos del contexto que van más allá de la acumulación de ciertos perfiles sociales en el territorio. Los resultados de nuestra investigación demuestran que esta influencia existe y que tiene una importancia que puede medirse. Incluso tras controlar las principales variables individuales y de hogar (que sabemos que están desigualmente distribuidas entre lo urbano y lo rural), vivir en un área metropolitana sigue aumentando ligeramente la probabilidad de participar en la movilidad intramunicipal y, de forma muy acentuada, la de ser parte de la movilidad intermunicipal.

Además, estos factores se manifiestan a diferentes escalas, pero la más relevante para las decisiones de movilidad en el área metropolitana es el mercado local de vivienda. Las áreas metropolitanas son una realidad funcional, pero en muchos casos se desestiman como unidad de análisis en favor del municipio, debido a la mayor disponibilidad de datos. Nuestro análisis pone de manifiesto, de manera similar a estudios previos (Kim et al., 2015; Van der Vlist et al., 2002; Dieleman et al., 2000; Cadwallader, 1989), cómo las dinámicas internas, la composición sociodemográfica de la población y el parque de viviendas existente explican la movilidad residencial metropolitana. Se ha comprobado también que existen diferencias sustanciales entre la movilidad intramunicipal y la intermunicipal. Las variaciones en la primera se pueden explicar por características referentes al dinamismo del conjunto metropolitano (como la presencia de extranjeros, el crecimiento de la población y la proporción de ocupados), mientras la segunda, que es además la forma de movilidad más característica de las áreas metropolitanas, se relaciona con otras variables, como la composición social y demográfica de la población o la estructura del parque de viviendas. Curiosamente, una vez controlado el resto de variables, las áreas más extensas no tienen una mayor movilidad intermunicipal. En conjunto, la 
movilidad intramunicipal parece ser más fácilmente explicable, debido a que los factores que la causan, tanto en los municipios de la cabecera como en los de la corona, son los mismos. Sin embargo, la movilidad intermunicipal es más compleja, ya que tanto las características de las áreas que le afectan como la diversidad de dinámicas que la componen (suburbanización, centralización y movilidad entre municipios de la corona) provocan que su explicación esté sujeta a factores contextuales (Feria, 2010) y coyunturales (Feria y Andújar, 2015). La dinámica intermunicipal mayoritaria, la suburbanización, es la que mejor puede predecirse. Los movimientos de salida hacia la corona parecen estar ligados a la saturación del mercado de vivienda de una ciudad central cada vez más envejecida (Galiana y Vinuesa, 2012) y con un perfil social medio-alto (López-Gay y Recaño, 2008). Contrariamente a la extendida idea de una suburbanización electiva y de clase media, típica de la configuración metropolitana norteamericana, en las ciudades españolas este modelo - que existe- convive con una suburbanización de clases populares. Sin llegar a la suburbanización de la pobreza que algunos autores describen (Covington, 2015; Cooke y Denton, 2015), el panorama es lo suficientemente complejo como para merecer ulteriores investigaciones.

Parte de lo que ocurre en una ciudad metropolitana solo cobra sentido en el marco de esa misma unidad de significado. La jerarquización de espacios, la imagen social de los entornos, la percepción del mercado residencial, etc. Cada área metropolitana es un mundo, con una historia y unas reglas propias. Pero, al mismo tiempo, todos esos mundos interaccionan en regiones urbanas más amplias y complejas, lo que genera regularidades. Una geografía compleja de espacios dinámicos —esferas que giran dentro de otras esferas - , en la que los individuos se trasladan o se asientan, sin muchas veces percibir que incluso al quedarse quietos pueden estar cambiando, puesto que, en ocasiones, es la ciudad la que se desplaza.

\section{Referencias bibliográficas}

Abrahamson, Marianne; BorgeGaR, Lars-Erik y Fransson, Urban (2000). «Housing Careers: Inmigrants on Local Swedish Housing Markets». Housing Studies [en línea], 17 (3), 11-13. <http://dx.doi.org/10.1080/02673030220134944>.

ARAgonés TAPIA, Juan Ignacio y AmÉRIgo Cuervo-Arango, María (1987). «Movilidad residencial en la ciudad: Factores determinantes y consecuencias». Estudios sobre Consumo [en línea], 11, 7.

Arévalo, Raquel; Ferrero, Dolores; Otero Giráldez, M.a Soledad y Uña ÁlvaREZ, Jacobo de (2008). Movilidad residencial en España: Un análisis longitudinal. Universidade de Vigo. Departamento de Economía Aplicada. Documentos de Traballo 0803.

Ault, Richard W.; Jackson, John D. y SABA, Richard P. (1994). «The Effect of Long-term Rent Control on Tenant Mobility». Journal of Urban Economics [en línea], 35 (2), 140-158.

$<$ http://dx.doi.org/10.1006/juec.1994.1009>. 
BAYONA-I-CARRASCO, Jordi y LÓPEZ-GAY, Antonio (2011). "Concentración, segregación y movilidad residencial de los extranjeros en Barcelona». Documents d'Anàlisi Geogràfica [en línea], 57 (3), 381-412. <http://dx.doi.org/10.5565/rev/dag.234>.

BAyOnA-I-CARrasco, Jordi y PujADAS-I-Rúbies, Isabel (2010). "Cambios residenciales internos en la ciudad de Barcelona: Evolución y características territoriales». Investigaciones Geográficas [en línea], 52, 9-36. <http://dx.doi.org/10.14198/INGEO2010.52.01>.

- (2014). «Movilidad residencial y redistribución de la población metropolitana: Los casos de Madrid y Barcelona». EURE [en línea], 40 (119), 261-287. <http://dx.doi.org/10.4067/S0250-71612014000100012>.

BERICAT, Eduardo (1994). Sociología de la movilidad espacial: El sedentarismo nómada. Madrid: Centro de Investigaciones Sociológicas / Siglo XXI.

Brown, Lawrence A. y Moore, Eric G. (1970). «The Intra-urban Migration Process: A Perspective». Geografiska Annaler: Series B. Human Geography [en línea], 52 (1), 1-13. <http://dx.doi.org/10.2307/490436>.

Cadwallader, Martin (1989). "A Synthesis of Macro and Micro Approaches to Explaining Migration: Evidence from Inter-State Migration in the United States». Geografiska Annaler: Series B. Human Geography [en línea], 85-94. <http://dx.doi.org/10.2307/490517>.

- (1992). Migration and Residential Mobility: Macro and Micro Approaches. Madison: University of Wisconsin Press.

Champion, Tony (2001). «Urbanization, suburbanization, counterurbanization and reurbanization». En: PADDISON, Ronan (ed.). Handbook of urban studies [en línea]. Londres: SAGE Publications. <https://doi.org/10.4135/9781848608375.n9>.

Cheshire, Paul (1995). "A new phase of urban development in Western Europe?: The evidence for the 1980s». Urban Studies [en línea], 32 (7), 1045-1063. <http://dx.doi.org/10.1080/00420989550012564>.

Clark, William A.V. (2013). "Life Course Events and Residential Change: Unpacking Age Effects on the Probability of Moving». Journal of Population Research [en línea], 30 (4), 319-334. <http://dx.doi.org/10.1007/s12546-013-9116-y>.

Clark, William A.V.; Duque-Calvache, Ricardo y Palomares-Linares, Isabel (2015). «Place Attachment and the Decision to Stay in the Neighbourhood». Population, Space and Place [en línea]. $<$ http://dx.doi.org/10.1002/psp.2001>.

Clark, William A.V. y Heskin, Allan D. (1982). "The Impact of Rent Control on Tenure Discounts and Residential Mobility». Land Economics [en línea], 58 (1), $109-117$. <http://dx.doi.org/10.2307/3146080>.

Clark, William A.V.y Huang, Youquin (2003). "The Life Course and Residential Mobility in British Housing Markets». Environment and Planning A [en línea], 35, 323-339. $<$ http://dx.doi.org/10.1068/a3542>.

Clark, William A.V. y Mulder, Clara (2000). "Leaving Home and Entering the Housing Market». Environment and Planning A [en línea], 32 (9), 1657-1671. <http://dx.doi.org/10.1068/a3315>. 
Clark, William A.V. y OnaKa, Jun L. (1982). "Life cycle and housing adjustment as explanations of residential mobility». Urban Studies [en línea], 20 (1), 47-57. <http://dx.doi.org/10.1080/00420988320080041>.

Clifford, William B.; Heaton, Tim y Fuguitt, Glenn V. (1982). «Residential Mobility and Living Arrangements among the Elderly: Changing Patterns in Metropolitan and Nonmetropolitan Areas». The International Journal of Aging and Human Development [en línea], 14 (2), 139-156. <http://dx.doi.org/10.2190/fh8g-m1lh-a5nm-64x1>.

CONTRERAS Gatica, Yasna (2011). "La recuperación urbana y residencial del centro de Santiago: Nuevos habitantes, cambios socioespaciales significativos». Eure [en línea], 37 (112), 89-113. <http://dx.doi.org/10.4067/S0250-71612011000300005>.

CoOke, Thomas J. y Denton, Curtis (2015). "The suburbanization of poverty?: An alternative perspective». Urban Geography [en línea], 36 (2), 300-313. <http://dx.doi.org/10.1080/02723638.2014.973224>.

COUlTER, Rory; HaM, Marteen van y FindLAY, Allan (2016). «Re-thinking residential mobility Linking lives through time and space». Progress in Human Geography [en línea], 40 (3), 352-374. <http://dx.doi.org/10.1177/0309132515575417>.

CourgeAu, Daniel (1988). «Méthodes de mesure de la mobilité spatiale: Migrations internes, mobilité temporaire et navettes. Présentation d'un Manuel de l'INED». Population [en línea], 43 (4), 877-880. <http://dx.doi.org/10.2307/1533496>.

Covington, Kenya L. (2015). «Poverty suburbanization: Theoretical insights and empirical analyses». Social Inclusion [en línea], 3 (2). <http://dx.doi.org/10.17645/si.v3i2.120>.

Dieleman, Frans M. (2001). «Modelling Residential Mobility: A Review of Recent Trends in Research". Journal of Housing and the Built Environment [en línea], 16 (3), 249-265. <http://dx.doi.org/10.1023/A:1012515709292>.

Dieleman, Frans M.; Clark, William A.V. y Deurloo, Marinus C. (2000). "The Geography of Residential Turnover in Twenty-seven Large US Metropolitan Housing Markets, 1985-95». Urban Studies [en línea], 37 (2), 223-245. <http://dx.doi.org/10.1080/0042098002168>.

DíEZ-Nicolás, Juan y Alvira, Francisco (dir.) (1985). Movimientos de población en áreas urbanas españolas. Madrid: Centro de Estudios de Ordenación del Territorio y Medio Ambiente.

DUQUE-CALVACHE, Ricardo (2015). La movilidad residencial y su relación con la vivienda: Áreas metropolitanas andaluzas. Sevilla: Consejería de Fomento y Vivienda. Junta de Andalucía.

Escobar-Mercado, Modesto; Fernández-Macías, Enrique y Bernardi, Fabrizio (2009). Análisis de datos con Stata. Madrid: Centro de Investigaciones Sociológicas. Serie Cuadernos Metodológicos.

FERIA, José María (2008a). «Un ensayo metodológico de definición de las áreas metropolitanas en España a partir de la variable residencia-trabajo». Investigaciones Geográficas [en línea], 46, 49-68. <http://dx.doi.org/10.14198/INGEO2008.46.03>.

- (2010). «La delimitación y organización espacial de las áreas metropolitanas españolas: Una perspectiva desde la movilidad residencia-trabajo». Ciudad y Territorio: Estudios Territoriales, 164, 208. 
- (2015). Definición y pautas generales de dinámica y organización espacial: Áreas metropolitanas andaluzas. Sevilla: Consejería de Fomento y Vivienda. Junta de Andalucía.

- (coord.) (2008b). Migraciones y movilidad residencial en Andalucía. Sevilla: Instituto de Estadística de Andalucía.

FERIA, José María y ANDÚJAR, Andrea (2015). «Movilidad residencial metropolitana y crisis inmobiliaria». Anales de Geografía [en línea], 35 (1), 13-40. <http://dx.doi.org/10.5209/rev_AGUC.2015.v35.n1.48962>.

GALIANA, Luis y VinUESA, Julio (2012). «Descentralización y recentralización en espacios metropolitanos maduros: El caso de Madrid». En: PAlacios, Antonio J. y PORRAS, David (eds.). Metrópolis: Dinámicas urbanas. Madrid: Universidad Autónoma de Madrid y Universidad Nacional de Luján.

GuTIÉRREZ-PUEBLA, Javier (1992). «La movilidad en Madrid: La configuración espacial de los flujos». Economía y Sociedad, 6, 99-122.

HanusCHeK, Eric A. y QuigleY, John M. (1978). «An Explicit Model of Intra-metropolitan Mobility». Land Economics [en línea], 54 (4), 411-429. <http://dx.doi.org/10.2307/3146168>.

JimÉNEZ-BlASCO, Beatriz (1989). "La movilidad residencial intraurbana». Anales de Geografía de la Universidad Complutense, 9, 271-277.

KIM, Jae Hong (2014). «Residential and Job Mobility: Interregional Variation and Their Interplay in US Metropolitan Areas». Urban Studies [en línea], 51 (13) 2863-2879. <http://dx.doi.org/10.1177/0042098013514496>.

Kim, Hyun; Woosnam, Kyle M.; Marcouiller, David W.; Aleshinloye, Kayode D. y CHOI, Yeol (2015). «Residential Mobility, Urban Preference, and Human Settlement: A South Korean Case Study». Habitat International [en línea], 49, 497-507. <http://dx.doi.org/10.1016/j.habitatint.2015.07.003>.

LÓPEZ-GAY, Antonio y RECAÑO, Joaquín (2008). «La renovación sociodemográfica de un centro urbano maduro: Perfiles migratorios y filtros residenciales en la ciudad de Barcelona». Scripta Nova: Revista Electronica de Geografia y Ciencias Sociales, 12, 126.

LU, Maixin (1998). "Analyzing Migration Decision Making: Relationships between Residential Satisfaction, Mobility Intensions, and Moving Behavior». Environment and Planning $A$ [en línea], 30 (8), 1473-1495. $<\mathrm{http} / / / \mathrm{dx}$.doi.org/10.1068/a301473>.

MalpezZI, Sthepen; Chun, Gregory H. y GreEn, Richard K. (1998). «New Place-toPlace Housing Price Indexes for US Metropolitan Areas, and Their Determinants». Real Estate Economics [en línea], 26 (2), 235-274. <http://dx.doi.org/10.1111/1540-6229.00745>.

MódenES-CABRERIZO, Juan Antonio (1998). Flujos espaciales e itinerarios biográficos: La movilidad residencial en el área de Barcelona. Departamento de Geografía. Universidad Autónoma de Barcelona. Tesis doctoral.

- (2008). «Movilidad espacial, habitantes y lugares: Retos conceptuales metodológicos para la geo-demografía». Estudios Geográficos [en línea], 69 (264), 157-178. <http://dx.doi.org/10.3989/egeogr.2008.i264.83>.

Módenes-CABrerizo, Juan Antonio y LÓPEZ-ColÁs, Julián (2004). «Movilidad residencial, trabajo y vivienda en Europa». Scripta Nova: Revista Electrónica de Geografía y Ciencias Sociales, 8, 159.

Mulder, Clara H. (1993). Migration dynamics: A life course approach. Amsterdam: Thesis Publishers. 
Munch, Jakob R. y Svarer, Michael (2002). «Rent Control and Tenancy Duration». Journal of Urban Economics [en línea], 52 (3), 542-560. <http://dx.doi.org/10.1016/S0094-1190(02)00502-8>.

Musterd, Sako; GenT, Wouter van; DAS, Marjolijn y LATTEN, Jan (2016). «Adaptive behaviour in urban space: Residential mobility in response to social distance». Urban Studies [en línea], 53 (2), 227-246. <http://dx.doi.org/10.1177/0042098014562344>.

Pendakur, Ravi y YOUnG, Nathan (2013). «Putting on the moves: Individual Household, and Community-level Determinants of Residential Mobility in Canada». Demographic Research [en línea], 28 (29), 767-796. <http://dx.doi.org/10.4054/DemRes.2013.29.28>.

Plantinga, Andrew J.; Détang-Dessendre, Cécile; Hunt, Gary L. y Piguet, Virginie (2013). «Housing Prices and Inter-urban Migration». Regional Science and Urban Economics [en línea], 43 (2), 296-306. <http://dx.doi.org/10.1016/j.regsciurbeco.2012.07.009>.

PUJADAS-I-RÚBIES, Isabel (2009). «Movilidad residencial y expansión urbana en la Región Metropolitana de Barcelona, 1982-2005». Scripta Nova: Revista Electrónica de Geografia y Ciencias Sociales, 13, 290.

Quigley, John M. y Weinberg, Daniel H. (1977). «Intra-urban Residential Mobility: A Review and Synthesis». International Regional Science Review [en línea], 2 (1), 41-66. <http://dx.doi.org/10.1177/016001767700200104>.

Rossi, Peter H. (1955). Why families move: A studie in the social psychology of urban residential mobility. Nueva York: Free Press of Glencoe.

Strassman, W. Paul (2000). «Mobility and Affordability in US Housing». Urban Studies [en línea], 37 (1), 113-126. <http://dx.doi.org/10.1080/0042098002311>.

Susino, Joaquín (2003). Movilidad residencial: Procesos demográficos, estrategias familiares y estructura social. Granada: Universidad de Granada. Tesis doctoral inédita.

Susino, Joaquín y DUQUe-CALVACHE, Ricardo (2013). «Veinte años de suburbanización en España (1981-2001): El perfil de sus protagonistas». Documents d'Anàlisi Geogràfica [en línea], 59 (2), 265-290. <http://dx.doi.org/10.5565/rev/dag.31>.

Vlist, Arno J. van der; Gorter, Cees; Nijkamp, Peter y Rietveld, Piet (2002). "Residential Mobility and Local Housing-market Differences». Environment and Planning $A$ [en línea], 34 (7), 1147-1164. <http://dx.doi.org/10.1068/a34176>. 


\section{Anexos}

Anexo 1. Modelo de regresión logística multinomial para la explicación de la movilidad intramunicipal e intermunicipal sin la variable residía en áreas metropolitanas

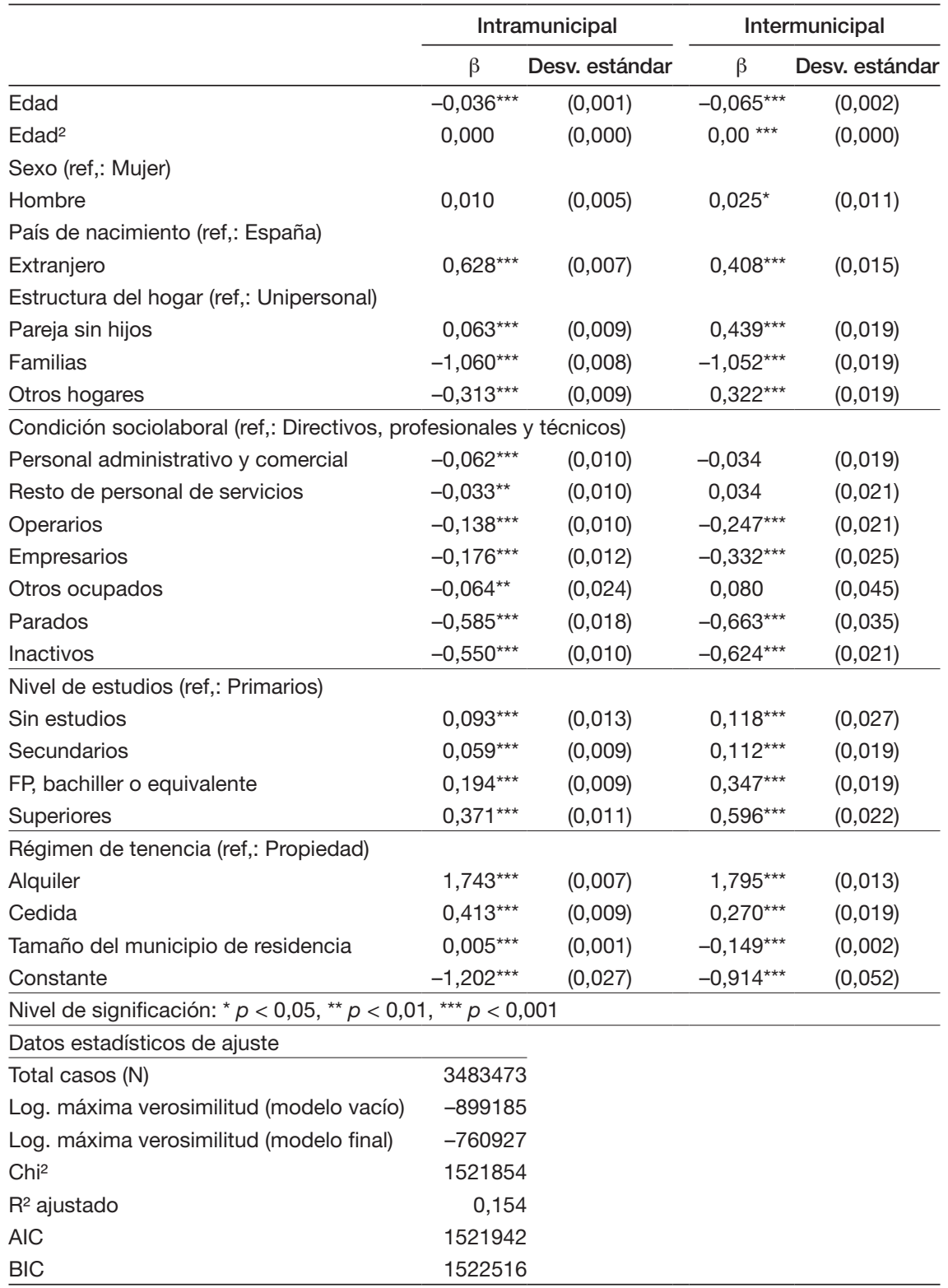

Fuente: elaboración propia a partir de los datos del INE, Censo 2011. 
Anexo 2. Cambio en los datos estadísticos de ajuste de los modelos de regresión logística multinomial para la explicación de la movilidad intramunicipal e intermunicipal

\begin{tabular}{lccc}
\hline \multicolumn{1}{c}{ Datos estadísticos de ajuste } & $\begin{array}{c}\text { Modelo sin } \\
\text { Residía en área } \\
\text { metropolitana }\end{array}$ & Modelo final & Diferencias \\
\hline Total casos $(N)$ & 3483473 & 3483473 & 0 \\
Log. máxima verosimilitud (modelo vacío) & -899185 & -899185 & 0 \\
Log. máxima verosimilitud (modelo final) & -760927 & -757858 & 3069 \\
Chi $^{2}$ & 1521854 & 1515716 & -6138 \\
$\mathrm{R}^{2}$ ajustado & 0,154 & 0,157 & 0,003 \\
$\mathrm{AIC}$ & 1521942 & 1515808 & -6134 \\
$\mathrm{BIC}$ & 1522516 & 1516409 & -6108 \\
\hline
\end{tabular}

Fuente: elaboración propia a partir de los datos del INE, Censo 2011.

Anexo 3. Análisis de casos atípicos para la movilidad intramunicipal metropolitana

\begin{tabular}{|c|c|c|c|c|c|}
\hline Caso/Área & $\begin{array}{c} \pm 1.96 \\
\text { desviaciones } \\
\text { estándar } \\
\text { respecto a lax }\end{array}$ & $\begin{array}{c}\text { Atípicos fuera } \\
\text { del diagrama } \\
\text { de caja }\end{array}$ & $\begin{array}{c}\text { Alta carga y } \\
\text { altos residuos }\end{array}$ & $\begin{array}{c}\text { Alta } \\
\text { contribución } \\
\text { a coeficientes } \\
\text { de las Vis } \\
\text { (dfbetas) }\end{array}$ & $\begin{array}{c}\text { Alta } \\
\text { contribución } \\
\text { al modelo } \\
\text { (Welsch, Dfits } \\
\text { o Cook) }\end{array}$ \\
\hline Arona-Tenerife Sur & Sí & Sí & Sí & Sí & Sí \\
\hline Jaén & Sí & Sí & Sí & No & Sí \\
\hline Vitoria & Sí & No & Sí & Sí & Sí \\
\hline Benidorm & No & No & Sí & No & Sí \\
\hline
\end{tabular}

Fuente: elaboración propia a partir de los datos del INE, Censo 2011. 